dan. Zwar ist die Einführung in erster Linie fürs Heimpublikum und die Befriedung der NIF gedacht - in Khartum werden öffentlichkeitswirksam tausende Flaschen Whisky in den Nil geschüttet (Warburg, 1990: 624) -, doch repräsentiert es den Tropfen, der den Friedensprozess im Süden zusammenbrechen lässt. Auch bescheren diese Beschlüsse der wenige Monate zuvor gegründeten Sudan People's Liberation Army (SPLA) jenen Zulauf aus Dinka und Nuer-Gemeinschaften, den sie für die ernsthafte Aufnahme des bewaffneten Kampfes benötigt.

\title{
Diskrepanzen des Widerstandes
}

Die SPLA und die mit ihr verbundene politische Bewegung, SPLM, sind aber keineswegs die erste Organisation, die den bewaffneten Kampf nach dem Addis Ababa Agreement wieder aufnimmt. Schon im März 1975 hatten sich ehemalige Militante aus den Anya-nya, vor allem Vertreter in der AutonomieRegierung marginalisierter Nuer-Gemeinschaften, als Anya-nya II zusammengefunden. Der Gründungsmythos der Anya-nya II geht auf einen Aufstand in der Garnison in Akobo an der äthiopischen Grenze zurück. Von Beginn an positioniert sich die Bewegung in direktem Widerspruch zum Addis Abeba Friedensvertrag und der südlichen Autonomie und erhebt die Forderung nach staatlicher Unabhängigkeit (LeRiche und Arnold, 2013: 31). Die Geschichte der Anya-nya II ist mit dem Osten der Region verbunden und entwickelt sich von Beginn an im Widerstand gegen die so wahrgenommene politische Dominanz der Bahr el-Ghazal Dinka aus dem Westen.

Diese Rivalität wird später zu blutigen bewaffneten Auseinandersetzungen mit der SPLM/A führen, die in die ab den späten 1980er-Jahren aufbrechenden ethnopolitischen Spannungen innerhalb der Organisation hineinspielen (Jok, 2017). Nicht nur steht die politische Positionierung der Anyanya II im Gegensatz zu der ambivalent vertretenen gesamtstaatlichen Orientierung der ursprünglichen SPLM/A. Auch ist die Anya-nya II in ihrer Zusammensetzung von Militanten aus dem Osten des Landes, hauptsächlich Nuer, dominiert. Die Spannungen spiegeln sich auf Führungsebene wider. Die Anya-nya II-Spitze, bestehend aus dem Nuer-Kommandanten William Abdallah Cuol, einem Nuer-Minister der Autonomie-Regierung, Samuel Gai Tut und dem Twic Dinka Akwot Atem de Mayen, einem langjährigen Gegner der Autonomieregelung, sieht sich nicht nur als historische Führung des Widerstandes legitimiert. Auch fühlt sie sich aufgrund ihres Alters und ihrer 
Kampferfahrung dem SPLM/A-Gründer John Garang moralisch übergeordnet.

John Garang de Mabior stammt wie Akwot Atem aus Twic County in Upper Nile. Zunächst entscheidet er sich mit finanzieller Hilfe von Verwandten für eine solide Ausbildung. Nach dem Schulbesuch in Tansania sichert sich Garang ein Stipendium für ein Bachelor-Studium in Ökonomie in den USA, das er erfolgreich abschließt (vgl. Natsios, 2012: 61-65). Danach kehrt er für weitere Studien nach Tansania zurück. An der University of Dar es Salaam beginnt er sein politisches Engagement. Wenig später schließt er sich den Anya-nya an und wird, im Jahr 1970, von Anya-nya-Führer Murtat Mayen zur militärischen Ausbildung nach Israel geschickt. Im Zuge der Integration der Anya-nya in die SAF im Gefolge des Addis Ababa Agreement wird Garang professioneller Soldat in der sudanesischen Armee. Diese Rolle nutzt er für eine weitere fundierte Ausbildung in den USA, zunächst militärisch, in einem Programm für Infanterieoffiziere in Fort Benning, Georgia, und später zivil. Er unterbricht seine militärische Karriere, um einen Masters-Abschluss in Agrarökonomie in Iowa zu erwerben.

Danach kehrt Garang zu den SAF zurück, wo er sich sofort, zunächst von Khartum aus, der Organisation einer südlichen Aufstandsbewegung unter den reintegrierten Anya-nya-Kämpfer:innen widmet. Als im Mai 1983 das 105. Bataillon der SAF in der Garnison in Bor in Jonglei rebelliert, überzeugt er seine sudanesischen Führungsoffiziere, zur Befriedung des Aufstandes in den Süden aufzubrechen. Er macht sich allerdings auf den Weg auf die äthiopische Seite der Grenze in Jonglei, wo er mit den Aufständischen zusammentrifft, die der Gegenoffensive der SAF auf Bor ausgewichen waren. Überhaupt entfaltet die Rebellion in Bor, zusammen mit kleineren Aufständen in Pochalla und Ayud, eine weitreichende Signalwirkung. "In response to a call to arms South Sudanese of all walks of life trekked all the way to the Ethiopian border where the first SPLM/A camps were established.« (Nyaba, 2000: 28)

Der Aufstand in Bor folgt unmittelbar auf den durch Kompromisse schwer erkauften Wahlsieg Nimeiris. Er ist im Kontext der sich zusehends manifestierenden Machtübernahme islamistischer Kräfte zu sehen. Zugleich ist die Aktion sorgsam vorbereitet. Kerubino Kuanyin Bol, wie Garang ein Twic Dinka, kommandiert die Rebellion und feuert dabei der Legende nach - die Anya-nya II waren ja schon Jahre zuvor aktiv - den ersten Schuss des zweiten sudanesischen Bürgerkrieges ab. Der Nuer-Major William Nyuon Bany Machar kommandiert parallel weitere Aufstände in kleineren Kasernen im Süden. Beide Gruppen begeben sich zur äthiopischen Grenze, wo sie mit John 
Garang zusammentreffen. Hier erfolgt die formelle Gründung der SPLM/A. Garang wird zum Anführer der neuen Bewegung ernannt, nicht zuletzt aufgrund seines militärischen und akademischen Leistungsausweises und seiner Fähigkeit zur Garantie der äthiopischen Unterstützung. In der KommandoKette folgen Kerubino Bol, William Nyuon und Salva Kiir Mayardit.

Am 31. Juli 1983 verkündet Garang die Gründung der SPLM/A, wobei zu diesem Zeitpunkt bereits 3.000 Mann unter seinem Kommando stehen. Mit der Gründung veröffentlicht die SPLM/A ihr erstes Manifest, das sich zu einer gesamtsudanesischen Revolution bekennt und dem Separatismus eine Absage erteilt. Ideologisch ist das Programm prononciert sozialistisch. Im Ton einer klassisch antiimperialistischen Befreiungsbewegung fordert die SPLM/A einen "protracted armed struggle in order to establish a Socialist system in the whole of Sudan« (Scott: 1985, 71). Die sozialistische Orientierung reflektiert eine Neuinterpretation der eigenen Position zum Kolonialismus, die auf einer kritischen Aufarbeitung der Anya-nya-Erfahrungen beruht: »1. [...] It is the colonial policy of divide and rule and the mechanics of peripheral development in the Sudan that are mainly responsible for the post-independence crises in the country. 2. [...] The transformation from direct colonialism to indirect colonialism.«

Der klarste Ausdruck der nun deklariert revolutionären Marschrichtung findet sich in Artikel 21 des Manifests. Dieser Artikel bringt den Gehalt der sogenannten »New Sudan Vision« auf den Punkt. Zugleich hebt er die SPLM/A markant von Anya-nya und, von unmittelbarer praktischer Relevanz, der parallel operierenden Anya-nya II ab.

"21. The immediate task of the SPLA/SPLM is to transform the Southern Movement from a reactionary movement led by reactionaries and concerned only with the South, jobs and self interest to a progressive movement led by revolutionaries and dedicated to the socialist transformation of the whole country. It must be reiterated that the principal objective of the SPLA/SPLM is not separation for the South. The South is an integral and inseparable part of the Sudan. Africa has been fragmented sufficiently enough by colonialism and neo-colonialism and its further fragmentation can only be in the interests of her enemies. «

Für die von Beginn an essenzielle äthiopische Unterstützung erweist sich die neue politische Positionierung als vorteilhaft. Im September 1974 hatten sozialistisch orientierte Militärs den äthiopischen Kaiser Haile Selassie gestürzt und eine Junta unter Leitung eines Provisorischen Militärverwaltungs- 
rats (amharisch Derg für »Council«) eingerichtet. Im Februar 1977 übernimmt Mengistu Haile Mariam die Führung der Derg, was deren sozialistische Orientierung nochmals verstärkt und zu einer Vertiefung der Beziehung mit dem ebenfalls sozialistisch orientierten Garang beiträgt. Garang kann dieses Netzwerk von Anfang an für den Aufbau der SPLM/A nutzen, was ein wesentlicher Faktor in der langjährigen Absicherung seiner eigenen Machtposition in der Bewegung ist.

Wenngleich das Derg-Regime einen Sturz des mit den USA verbündeten Nimeiri herbeiführen will, steht es separatistischen Bewegungen prinzipiell ablehnend gegenüber (Johnson, 2016a: 62). Dies hat seinen Grund in der fragilen Situation im eigenen Land. Die von Garang forcierte »New Sudan«-Orientierung ist zum Teil aus dieser Grundbedingung der äthiopischen Partner zu erklären. Im Nachhinein zeigt sich, dass die Derg die separatistische Herausforderung richtig eingeschätzt hatten. Das im Jahr 1993 seine Unabhängigkeit von Äthiopien erlangende Eritrea ist (neben dem politisch besonders gelagerten Fall von Namibia ${ }^{1}$ ) die einzige nationale Sezessionsbewegung im postkolonialen Afrika, die eine Eigenstaatlichkeit erringen kann - bis zur Unabhängigkeit des Südsudan im Jahr 2011.

Die neue regionale Konfiguration verändert die geopolitische Verortung des Konfliktes signifikant. War die Anya-nya noch von Israel gegen die partiell kommunistische Gefahr im Sudan und Ägypten unterstützt worden, gilt nun das sudanesische Regime als Verbündeter der USA und der südsudanesische Widerstand in seiner dominierenden Fraktion als sozialistisch geprägt. Dies verstrickt den Konflikt in den Kontext der Systemauseinandersetzung des Kalten Krieges. Die Kalter-Kriegs-Logik steht im Kontrast zur pragmatischen Auslegung der »New Sudan«-Vision durch die SPLM/A, die sich alles andere als vehement sozialistisch artikuliert. »In sum, the SPLA/M's New Sudan was to be a secular democracy with devolved governance.« (LeRiche und Arnold, 2013: 35)

Die äthiopische Unterstützung gibt der SPLM/A die strategische Überhand gegenüber den Anya-nya II und anderen, kleineren Rebellenbewegungen. Dies löst eine gewisse Sogwirkung zu ihren Gunsten aus. Die beiden Organisationen unterscheiden sich jedoch nicht nur in der Zusammensetzung ihrer internationalen Unterstützung und ihrer politischen Positionierung. Garang tritt mit der SPLM/A an, erstmals eine einheitliche Formate aber gegen das Apartheit-Regime in Südafrika faktisch nicht durchgesetzt werden. 
tion zu schaffen, die mit der milizorientierten Ethnopolitik der Anya-nyaGeschichte zu brechen in der Lage ist. »The SPLA/M sought to be a focused, singular national front for change, making it substantively different from previous rebel armies.« (LeRiche und Arnold, 2013: 31)

Dieses Ziel ist nicht einfach umzusetzen. Garang verlässt sich in seinem Führungsstil auf enge Vertraute, oftmals Dinka, was dazu führt, dass die SPLM/A schnell als eine Dinka-Bewegung wahrgenommen wird. Dieses Vorurteil haftet der Organisation trotz der gezielten Durchmischung der Führungspositionen über die gesamte Dauer des Bürgerkrieges an. Das zum Teil brutale Vorgehen gegen Nuer-Gemeinschaften in ihrem Kampf gegen die Anya-nya II und die tendenzielle Marginalisierung von EquatoriaGemeinschaften, die die ursprüngliche Anya-nya dominiert hatten, ist der Popularität der SPLM/A ebenfalls nicht zuträglich. Das Zentrum der Kämpfe mit der in Upper Nile verwurzelten Anya-nya II liegt im Osten der Region. Die SPLM/A dominiert zwar militärisch, kann aber bis zum Jahr 1991, als der Nasir-Split die Karten in der politischen Konfiguration des südsudanesischen Widerstandes neu mischt, keinen entscheidenden Sieg für sich verbuchen.

Der Kampf der SPLM/A gegen die sudanesischen Truppen konzentriert sich anfänglich ebenfalls auf das äthiopische Grenzgebiet. Dabei gelingen schnell zusätzliche Rekrutierungen, insbesondere in Gemeinschaften, die in beständiger gewaltsamer Auseinandersetzung mit Baggara-Stämmen aus dem Norden leben. $\mathrm{Zu}$ einem zweiten Schwerpunkt der Operationen entwickelt sich Bahr el-Ghazal, die Heimat von Garang und Kerubino Bol. Die niederschwellige, aber beständige Auseinandersetzung mit gewaltsamen Kampagnen von Baggara befördert dort ebenfalls die Rekrutierung neuer Kämpfer:innen. Auch in den südlichen Provinzen dominiert die SPLA den Kampf gegen die SAF.

Angesichts des oftmals kompromisslosen Vorgehens gegen die Zivilbevölkerung und eines strengen Steuerregimes, das praktische Unterstützungsleistungen und Rekrutierungen einschließt, kann sich die SPLM/A dennoch nicht auf eine breite Beliebtheit abstützen. Ihre Anziehungskraft ist untrennbar verknüpft mit den rasch eintretenden, einschneidenden militärischen Erfolgen. Die Organisation wächst beständig und schnell. Zählt die SPLA am Ende des Jahres 1985 noch etwa 10.000 Kämpfer:innen, sind es Ende des Jahres 1989 bereits über 70.000. Diese Zahlen erinnern eher an eine konventionelle Armee denn an eine Guerillaorganisation (LeRiche und Arnold, 2013: 67). Später wird Garang diese Periode als die goldenen Jahre der Bewegung bezeichnen. Schon früh werden staatliche Infrastrukturprojekte angegriffen. In 
einer ihrer ersten Aktionen zerstört die SPLA den monumentalen Bagger »Lucy«, der die Aushebung des Jonglei-Kanals, einem Großprojekt zur Umleitung des Weißen Nil um den Sudd, durchführt. Die SPLA kann so die Einstellung dieses lange geplanten Unterfangens erzwingen, was sowohl den Nordsudan als auch Ägypten empfindlich trifft.

Nimeiri setzt in seinem Abwehrkampf um den Erhalt seiner Präsidentschaft immer stärker auf südsudanesische Stammesmilizen, die sich aus historischen Gründen im Konflikt mit der SPLM/A befinden. Eine solche Gruppe rekrutiert sich aus Murle in Jonglei, die sich in einer langjährigen Auseinandersetzung mit den in dieser Periode in der SPLM/A wichtigen Bor Dinka befinden. Mundari-Milizen spielen eine ähnliche Rolle in Central Equatoria. Doch erweisen sich diese Milizen als zu schlecht ausgerüstet und zu schwach, um die SPLM/A ernsthaft herauszufordern.

Die anwachsende Ölexploration im informellen Grenzgebiet zwischen Süden und Norden zwingt Nimeiri zudem zur Konzentration seiner Kräfte auf Greater Upper Nile, speziell auf die Region um Bentiu, wo sich die produktivsten Ölblocks befinden. In Greater Upper Nile kommt es zu einer Kooperation der SAF mit den Anya-nya II. Diese verfügen in ihrem Heimatterritorium nach wie vor über eine starke Präsenz. Allerdings sind sie gegen die übermächtige SPLM/A auf externe Unterstützung angewiesen, die nun vom vormaligen Erzfeind kommt. Dies führt zur paradoxen, im südsudanesischen Kontext aber nicht ungewöhnlichen Situation, dass die in ihren politischen Forderungen radikalere Anya-nya II, die für eine Loslösung des Südens eintritt, von Khartum gegen eine Kraft unterstützt wird, die diese Loslösung tendenziell ablehnt. Diese Konstellation wird sich wenige Jahre später in der Folge des Nasir-Splits wiederholen. Für die Anya-nya II ist die sudanesische Unterstützung langfristig allerdings nicht ausreichend. Sie gerät mehr und mehr in die Defensive und ihre historische Führung wird in Gefechten mit der SPLA getötet.

Unterdessen kommt es im Sudan zu einem Umsturz. Eine desaströse Hungersnot in Darfur von 1984 bis 1985, die auch Teile des Südens in Mitleidenschaft zieht, zerstört die letzten Teile der Unterstützung für das NimeiriRegime. Stark steigende Nahrungsmittelpreise führen zu Straßenprotesten in Khartum. Im April 1985 wird Nimeiri von einer von islamistischen Kräften unterstützten Gruppe von Militärs gestürzt. Nach der Machtübernahme verspricht die Militärregierung baldige Wahlen. Im April 1986 werden diese tatsächlich abgehalten - wiederum jedoch nicht im Süden, wo die weitgehen- 
de Dominanz der SPLM/A und die damit einhergehende, offiziell deklarierte »Unsicherheit« deren Abhaltung verhindert.

In Folge des Sieges seiner Umma Party wird Sadiq al-Mahdi erneut zum sudanesischen Premierminister ernannt. In dieser, seiner zweiten Amtsperiode hält er sich sogar über drei Jahre. An der militärischen Vorgangsweise im Süden ändert die zivile Regierung jedoch nichts. Al-Mahdi zeigt sich kompromisslos. Er setzt den Krieg mit voller Vehemenz fort, wobei er Nimeiris Strategie der Anwerbung verbündeter Stammesmilizen weiterführt, wenngleich mit wechselndem Erfolg. Von Zande-Milizen wird etwa berichtet, dass sie die nordsudanesische Unterstützung dankend annehmen, diese dann aber in Auseinandersetzungen im Grenzgebiet mit Milizen aus Zaire, der heutigen DRC, und keineswegs gegen die SPLM/A einsetzen.

Die SPLM/A ändert ab Mitte der 1980er-Jahre ihre Strategie. Nach einigen harten bewaffneten Kampagnen gegen die mit Khartum kooperierenden Milizen wird die Politik gegenüber nicht in die formalen SPLA-Strukturen eingegliederten bewaffneten Kräften offener und kooperativer. Auch startet die SPLM/A eine Kampagne zur Steigerung ihrer Popularität in der Landbevölkerung, just zu einer Zeit, als die sudanesische Regierung durch eine immer rücksichtslosere Vorgangsweise der SAF ihre letzten Sympathien verspielt (Johnson, 2016a: 83). Dies ermöglicht der SPLA strategische Initiativen auch in den nordwestlichen Regionen. Bahr el-Ghazal entwickelt sich zum neuen Zentrum der militärischen Operationen. Diese werden vornehmlich vom heutigen Präsidenten Salva Kiir kommandiert - John Garang selbst hält sich nur selten in der Region auf.

Im Jahr 1989 startet die SPLA ihre überaus erfolgreiche »Bright Star Campaign« (Madut-Arop, 2006: 189-222), im Zuge derer es ihr gelingt, zwei Drittel des Südsudan unter Kontrolle zu bringen. Noch im selben Jahr kontrolliert sie nach Übernahme einiger Garnisonen, etwa in Torit und Kapoeta, drei Provinzhauptstädte: Torit, Bor und Nasir. Juba befindet sich unter ihrer Belagerung. Zugleich zeitigt der Krieg einschneidende humanitäre Konsequenzen. Bereits 1988 kommt es in Bahr el-Ghazal zu einer Hungersnot, die 250.000 Tote fordert. Das humanitäre Versagen beantworten verschiedene UN-Agenturen und Hilfsorganisationen in Khartum mit einer Verstärkung ihrer Kooperation. Nach einer Einigung mit der sudanesischen Regierung und der SPLM/A erfolgt die Einrichtung einer strukturierten Hilfsoperation, die weite Teile des Sudan umfasst. Das markiert den Beginn der Operation Lifeline Sudan (OLS), die im April 1989, noch informell und ohne vertragliche Basis, ihre Tätigkeit aufnimmt. Die direkte Kooperation mit der SPLM/A führt um- 
gehend zu einer Aufteilung der OLS in einen nördlichen und einen südlichen Sektor, wobei der südliche Sektor in Abstimmung mit der SPLM/A - und nicht mit der sudanesischen Zentralregierung - organisiert wird. Diese Abstimmung geht mit zahlreichen Herausforderungen und Problemen einher. Beide Kriegsparteien versuchen, die Hilfe entlang militärtaktischer Erwägungen zu beeinflussen, was die OLS-Aktivitäten unvermeidlich politisiert.

Aus einer Situation der Stärke und der internationalen Anerkennung heraus bietet die SPLM/A in dieser Phase der Regierung al-Mahdi Friedensverhandlungen an, die diese auch annimmt. Doch ein erneuter politischer Umsturz verhindert die Umsetzung. Am 30. Juni 1989 putscht das sudanesische Militär mit massiver Unterstützung der NIF, diesmal unter Führung von Brigadier Omar Hassan al-Bashir, der zum Präsidenten ernannt wird. Er wird dieses Amt für fast drei Jahrzehnte ausüben und damit den Konflikt im Süden entscheidend prägen. Ab den späten 1990er-Jahren versucht Bashir, seine Herrschaft in eine zivile Form zu überführen. Im Jahr 1998 gründet er gemeinsam mit al-Turabi die National Congress Party (NCP), die sich nach der Absetzung Turabis in einem internen Coup am Jahresende 1999 zum Vehikel des sich intern zunehmend komplex und volatil konfigurierenden Systems Bashir entwickelt (Collins, 2008: 227). Im Unterschied zu vorherigen Regierungen nimmt Bashirs Regime, nicht zuletzt aufgrund des Drucks der NIF, auch die politisch-ideologische Herausforderung durch die SPLM/A an. Dies resultiert in einer Verschiebung der Kräftekonstellation.

Zunächst rückt das Bashir-Regime die Auseinandersetzung in einen explizit religiösen Fokus. Dies ist eine direkte Antwort auf die Bildung der National Democratic Alliance (NDA), in der sich neben den meisten ehemals legalen sudanesischen Parteien, etwa den Kommunisten, der Democratic Unionist Party (DUP) und der Umma Party, auch die SPLM/A einfindet. Dies ist insofern bemerkenswert, als sich die SPLM/A noch wenige Wochen zuvor mit einer von der Umma Party angeführten sudanesischen Regierung im Krieg befunden hatte. Eine weitere taktische Antwort auf diese für Bashir potenziell gefährliche politische Konstellation gibt das Regime mit einer "peace from within«Kampagne, die im Anschluss an das Verhandlungsangebot an al-Mahdi die Bereitschaft von Teilen der SPLM/A zum Abschluss eines Friedensvertrages erfühlen soll. Allerdings zielt "peace from within« nicht auf einen umfassenden Friedensvertrag, sondern auf eine Spaltung der Widerstandsbewegung ab. Die bis in die späten 1990er-Jahre laufende Kampagne (Young, 2012: 86) führt bald zu einer Annäherung zwischen der sudanesischen Regierung und 
einigen Unzufriedener in der SPLM/A-Führung, die sich von Garangs autoritärem Führungsstil an den Rand gedrängt fühlen.

\section{Vorboten eines Bürgerkrieges}

Garangs Position wird aber weniger vom Regierungswechsel im Sudan denn vom Regierungswechsel in Äthiopien erschüttert. In den späten 1980er-Jahren wird die Position des Derg-Regimes gegenüber verschiedenen Rebellenorganisationen, insbesondere aus dem nördlichen Tigray, prekär. Die SPLA kann sich einer unmittelbaren Verwicklung in diese Kämpfe nicht entziehen. SPLA-Kontingente unterstützen, nicht zuletzt aus einer Art historischer Bringschuld, den Abwehrkampf der Derg militärisch. Diese eindeutige politisch-militärische Positionierung führt nach dem endgültigen Sturz des Derg-Regimes im Mai $1991 \mathrm{zu}$ unmittelbar spürbaren negativen Einschnitten. Der Weg zu Verhandlungen mit den neuen Machthabern, der Rebellenallianz Ethiopian People's Revolutionary Democratic Front (EPDRF) unter dem neuen Präsidenten Meles Zenawi ist verbaut. Die militärische Allianz bricht zusammen. "Zenawi immediately cut off all arms shipments to Garang, shut down the SPLA bases in western Ethiopia, and ended the training and support programs. [...] The external base of support for Garang's war disappeared in a single moment.« (Natsios, 2012: 97)

Der Umsturz in Äthiopien führt auch zu humanitären Verwerfungen. In Gambela, der äthiopischen Region an der Grenze zu Südsudan, kommt es zu massiven Fluchtbewegungen, was zu einer Verschlechterung der Nahrungsmittelsituation in Jonglei auf der südsudanesischen Seite führt. Khartum verbietet OLS und dem in ihrem Rahmen operierenden World Food Programme (WFP), humanitäre Güter in dieser Kernregion des bewaffneten Widerstandes zu liefern. Dies setzt wiederum die SPLM/A unter beträchtlichen Handlungsdruck, insbesondere im konkreten Umgang mit den Geflüchteten.

Innerorganisatorisch wirkt sich der äthiopische Machtwechsel fatal aus. Garangs Führungsposition ist weniger aufgrund seine persönlichen Leadership-Qualitäten unumstritten. Sein Hang zu harschen und mitunter despotischen Entscheidungen wird von den meisten führenden Exponent:innen der Bewegung seit der Gründung der SPLM/A kritisiert (Rolandsen und Daly, 2016: 121). Es ist vielmehr seine persönliche Verbundenheit mit der für die Organisation lebenswichtigen äthiopischen Unterstützung, die Garangs Unantastbarkeit erklärt. Und diese Unterstützung geht nun verloren. »As 